\title{
The Na-O anticorrelation in horizontal branch stars
}

\section{NGC 2808}

\author{
R. G. Gratton ${ }^{1}$, S. Lucatello ${ }^{1}$, E. Carretta ${ }^{2}$, A. Bragaglia ${ }^{2}$, V. D’Orazi ${ }^{1}$, and Y. Al Momany ${ }^{1,3}$ \\ 1 INAF - Osservatorio Astronomico di Padova, Vicolo dell'Osservatorio 5, 35122 Padova, Italy \\ e-mail: raffaele.gratton@oapd.inaf.it \\ 2 INAF - Osservatorio Astronomico di Bologna, via Ranzani 1, 40127 Bologna, Italy \\ ${ }^{3}$ European Southern Observatory, Alonso de Cordova 3107, Vitacura, Santiago, Chile
}

Received 13 July 2011 / Accepted 12 September 2011

\section{ABSTRACT}

\begin{abstract}
Globular clusters have been recognized to host multiple stellar populations. A spectacular example of this is the massive cluster NGC 2808, where multiple populations have been found along the horizontal branch (HB) and the main sequence (MS). Studies of red giants showed that this cluster appears homogeneous insofar Fe abundance is concerned, but it shows an extended anticorrelation between $\mathrm{Na}$ and $\mathrm{O}$ abundances. The Na-poor, O-rich population can be identified with the red MS, and the Na-rich, O-poor one with the blue one. This may be understood in terms of different He content, He being correlated with Na. A prediction of this scenario is that He-rich, Na-rich He-core burning stars, because they are less massive, will end up on the bluer part of the HB, while He-poor, Na-poor stars will reside on the red HB. The aim of this paper is to verify this prediction. To this purpose, we acquired high-resolution spectra of regions including strong $\mathrm{O}$ and Na lines in several tens of HB stars of NGC 2808, sampling both the red and blue parts of the HB. We limited our analysis to those blue HB stars cooler than the gap at $11500 \mathrm{~K}$, because diffusion and radiative pressure are known to strongly modify the atmospheric composition of warmer stars. We indeed found a strict correspondence between the colour of the HB stars and their $\mathrm{Na}$ and $\mathrm{O}$ abundances: all blue HB stars are very O-poor and Na-rich. In addition, we found that while all the red HB stars are more O-rich and Na-poor than the blue ones, there is a moderate Na-O anticorrelation among them as well. This anticorrelation is in turn related to the colour of the red HB stars. These results reinforce the connection between Na and $\mathrm{O}$ abundances and the second parameter phenomenon, and show that there are more than three stellar populations in NGC 2808 because only a fraction of the red HB stars belong to the primordial population of this cluster.
\end{abstract}

Key words. stars: evolution - stars: abundances - stars: Population II - globular clusters: general

\section{Introduction}

In the recent years, it has become clear that the formation of the most massive stellar clusters, the globular clusters (GCs), is a complex phenomenon, involving several episodes of star formation, with the latest generations of stars forming from material polluted by the ejecta of a fraction of the earliest (primordial) population, as first suggested by Norris et al. (1981; see also Gratton et al. 2001, 2004; Carretta et al. 2010). Evidence for the presence of these different populations stems from their chemical composition, in particular the abundances of the light elements involved in $p$-capture processes $(\mathrm{C}, \mathrm{N}, \mathrm{O}, \mathrm{Na}, \mathrm{Mg}, \mathrm{Al}, \mathrm{Si}$ : Osborn 1971; see also Cottrell \& Da Costa 1981; Kraft 1994; Denisenkov \& Denisenkova 1989; Carretta et al. 2009a,b, and references therein), as well as from the splitting of sequences in the colour magnitude diagram (see e.g., Piotto 2008). While in the recent years some spectacular splitting of the main sequence (MS) have been found (see e.g., Piotto et al. 2007), the most dramatic evidence concerns the horizontal branch (HB) and was found already several decades ago (Harris 1974). This splitting of the $\mathrm{HB}$ has long been related to the second parameter problem (Sandage \& Wildey 1967; van den Bergh 1967) but it was only quite recently that a plausible cause has been identified

\footnotetext{
* Based on observations collected at ESO telescopes under programme 386.D-0086.
}

in variations of the He content related to the multiple populations (D'Antona \& Caloi 2004; but see Norris et al. 1981, for a very similar early suggestion). As pointed out by D'Antona et al. (2002), variations in the He content among different stellar generations in GCs are expected to be present, correlated to the variation of the abundances of the $p$-capture process elements. These He abundance variations are invoked to explain the multiple MSs seen in mono-metallic GCs like NGC 2808. However, because $\mathrm{He}$-rich stars are expected to burn $\mathrm{H}$ at a faster rate than the He-poorer ones while on the MS, their progeny on the HB is expected to be less massive, then bluer, than the progeny of He-normal stars. Carretta et al. (2007) and Gratton et al. (2010) discussed the general correlation between the abundances of $p$-capture elements and the colour of HB stars, and found indeed that a close relation exists between the extension of the $\mathrm{Na}-\mathrm{O}$ and $\mathrm{Mg}-\mathrm{Al}$ anticorrelations, and that of the $\mathrm{HB}$, precisely as expected in this interpretative scenario. These studies also showed that the main parameter driving the whole phenomenon of multiple populations is the cluster mass (confirming previous similar results obtained by Recio-Blanco et al. 2006; Carretta et al. 2006, 2009a), and that the second parameter can be explained almost entirely by a combination of variations of the age and mass of GCs.

While this is extremely intriguing, a definitive confirmation of this scenario with direct determination of the chemical 
composition of HB stars is required. The surface composition of the hottest stars on the HB, those with temperatures $>11500 \mathrm{~K}$ (the location of the so-called $u$-jump: Grundahl et al. 1999), are known to be heavily influenced by sedimentation caused by diffusion and by levitation through radiative pressure effects (see, e.g., Behr et al. 1999, 2003; see also Grundahl et al. 1999; Caloi 1999). However, the same studies revealed that abundances for cooler stars can be reliably determined, although the low $S / N$ and wavelength range of the spectra available at the epoch prevented an accurate determination of the abundances of those elements of interest here $(\mathrm{O}, \mathrm{Na})$. Behr et al. (2003) first obtained He abundances for cool BHB stars but the large error bars did not allow the authors to use them as diagnostics for the multiple population scenario. Very recently, a few investigations tried to measure the abundances of $\mathrm{O}$ and $\mathrm{Na}$, and even of $\mathrm{He}$, in such stars with sufficiently good accuracy. The pioneering study was the analysis of five blue HB stars in NGC 6752 by Villanova et al. (2009). This cluster has a very blue HB, and the observed stars (with temperatures $>11500 \mathrm{~K}$ ) are among the coolest HB stars. They behave as expected, most of them being Na-poor and O-rich, save for one star, which is more luminous than the others, and likely is an evolved object that probably started its HB evolution at a much higher temperature. Two other studies (Marino et al. 2011; and Villanova et al. 2011) focused on M 4, the nearest GC. This cluster has a much redder HB than NGC 6752, so that stars on both the red and blue sides of the RR Lyrae instability strip could be studied. Again, as expected, the red HB stars were found to be Na-poor and O-rich, and the blue ones Na-rich and $\mathrm{O}$-poor.

In this paper we present a similar investigation of the $\mathrm{HB}$ of NGC 2808. NGC 2808 is among the most massive Galactic GCs, and has become the most classical example of multiple populations. For a long time it was known to have a very peculiar $\mathrm{HB}$, with a multimodal distribution of colours and masses (Harris 1974; see Bedin et al. 2000; Momany et al. 2004; and Dalessandro et al. 2011, for recent accurate photometry). D'Antona et al. (2004, 2005) observed a widening of the MS, later found by Piotto et al. (2007) to consist of three distinct sequences, which can be explained with different values of the He content. D'Antona et al. (2004) correlated the different MSs with the different populations of $\mathrm{HB}$ stars. Low $S / N$, highresolution spectra along the BHB of NGC 2808 were obtained by Recio-Blanco et al. (2004) and were used to derive radial and rotational velocities. Pace et al. (2006) obtained intermediateresolution spectra for a number of $\mathrm{HB}$ stars, but they were only able to derive the Fe abundances from these spectra. They found that stars cooler than the $u$-jump at $\sim 12000 \mathrm{~K}$ provide values similar to those obtained for red giants, while metallicity increases with temperature for stars warmer than this limit. Carretta et al. (2004, 2006) studied the Na-O anticorrelation in several hundred red giants, and found an extended anticorrelation, with different peaks in the distribution. Bragaglia et al. (2010a) used this same observational material to derive evidence of the variation of the He content along the RGB, with results that on the whole agree with those obtained from the colours of MS and HB. However, while very suggestive, the connection between the chemical anomalies along the RGB and the multiple MS and HB could not be definitively shown by investigations that used very different techniques on stars in different evolutionary phases. To provide this definitive evidence, about one year ago, Bragaglia et al. (2010b) obtained spectra for one star on the blue MS, and another on the red one. They found that as expected, the blue MS star is $\mathrm{Na}$ - and $\mathrm{Al}$-rich and $\mathrm{Mg}$-poor, while the red one is $\mathrm{Na}$ - and $\mathrm{Al}$-poor and $\mathrm{Mg}$-rich. In this paper, we intend to complete the observational pattern by determining the abundances of $\mathrm{O}$ and $\mathrm{Na}$ for several tens of HB stars, using spectra acquired with FLAMES+GIRAFFE at VLT. We noticed immediately that while we possibly detected the He I line at $5876 \AA$ in the spectra of several of the BHB stars, stars are so faint that only prohibitively long exposure times would have ensured the $S / N$ required for accurate measure of the $\mathrm{He}$ abundance, as needed to distinguish primordial abundances from those expected for second generation stars in the multiple population scenarios ${ }^{1}$. Therefore we limited our analysis to $\mathrm{Na}$ and $\mathrm{O}$ abundances, for which the $S / N$ required to provide sensible results is much lower.

The structure of the paper is the following: in Sect. 2 we present the observational data; in Sect. 3 we explain our analysis methods; in Sect. 4 we discuss the Na-O anticorrelation; conclusions are drawn in Sect. 5.

\section{Observation}

We used the high multiplex capability of GIRAFFE at VLT (Pasquini et al. 2004) to acquire spectra for 49 stars along the red HB (RHB) and 36 on the blue HB (BHB) of NGC 2808. This was the maximum number of fibres that we were able to allocate to stars in these evolutionary phases given the practical limitations caused by the finite size of the fibre heads ${ }^{2}$. In addition, 34 fibres were used to point faint stars along the RGB (below the RGB bump), the remaining ones being used to acquire sky spectra. The median spectra from these last fibres were subtracted from those used for the stars. This was of particular relevance here, because the observed stars are typically very faint. Two spectral configurations were used, HR12 and HR19, providing high-resolution spectra including the strongest features of O I (the IR triplet at 7771-74 $\AA$ ) and Na I (the resonance D doublet at 5890-96 $\AA$, as well as the subordinate strong doublet at 8183-94 $\AA$ ) accessible from ground. The O I triplet and the $\mathrm{D}$ lines of $\mathrm{Na}$ I are the only lines of these two elements that can be used to determine $\mathrm{O}$ and $\mathrm{Na}$ abundances without a prohibitively observing time for NGC 2808. A few lines of $\mathrm{Mg}, \mathrm{Al}$, $\mathrm{Si}, \mathrm{Ca}, \mathrm{Fe}$, and $\mathrm{Ba}$ were also included in the selected observing ranges.

Our programme was executed in service mode, and not all planned observations were actually carried out, so that in total we had $1.5 \mathrm{~h}(2 \times 45 \mathrm{~min}$ exposures $)$ and $6.15 \mathrm{~h}(7 \times 53 \mathrm{~min})$ of observation with the gratings HR12 and HR19, respectively. The $S / N$ of the summed spectra is typically $\sim 20$ for the RHB stars. For the BHB stars, spectra with grating HR12 have $S / N \sim 15$ and those with grating HR19 $S / N \sim 10$. The spectra were reduced using the ESO FLAMES GIRAFFE pipeline version 2.8.7. Sky subtraction, combination of individual exposures for each star, translation to rest-frame and continuum tracing were performed within $\mathrm{IRAF}^{3}$. Telluric lines were removed from the longest wavelength spectra by subtracting the average

\footnotetext{
1 In practice, among stars with temperature $<11500 \mathrm{~K}$, the He I line is detected only for star \#32 324, and even in that case with such an error on the equivalent width that the estimate on He abundance is not useful. For the coolest stars the He line is not detectable in our noisy spectra even when they were summed together.

2 All stars were chosen to be free from any companion closer than 2 arcsec and brighter than $V+2 \mathrm{mag}$, where $V$ is the target magnitude. 3 IRAF is distributed by the National Optical Astronomical Observatory, which are operated by the Association of Universities for Research in Astronomy, under contract with the National Science Foundation.
} 

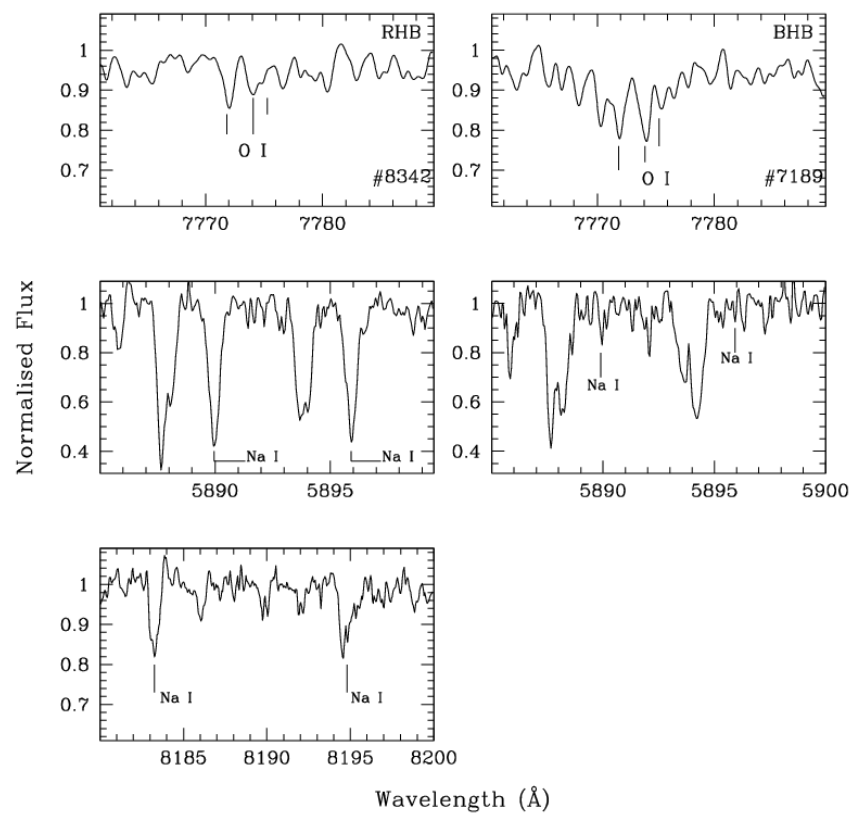

Fig. 1. Portions of the spectra of the RHB star \#8342 (left column) and of the BHB star \#7189 (right column). From top to bottom: the regions

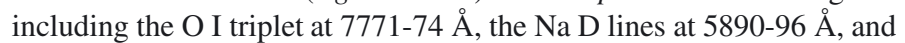
the Na doublet at 8183-94 $\AA$. The spectra in the region of the O I triplet have been smoothed by a Gaussian with $F W H M=0.3 \AA$. The Na D interstellar lines are clearly visible in the median panels.

spectrum of those BHB stars for which the observations have $S / N>8$. This combined spectrum has a $S / N \sim 50$ (much higher than the $S / N$ of the individual programme stars), and was obviously taken with the same airmass than the programme star, so that the excision of the telluric lines turned out to be excellent. Examples of spectra are shown in Fig. 1.

Figure 2 shows the location of the programme stars on the colour magnitude diagram of NGC 2808. Our NGC 2808 ground-based photometric catalogue (see Momany et al. 2004) consists of $U B V$ observations obtained at the Wide-Field Imager (WFI) mounted on the $2.2 \mathrm{~m}$ ESO-MPI (La Silla, Chile). The WFI catalogue has a total field of view of $34^{\prime} \times 33^{\prime}$. Photometric data for the programme stars are listed in Table 1 . The $K$ magnitudes are from the 2MASS point source catalogue (Skrutskie et al. 2006). Note that no information on membership of the programme stars to the cluster was available prior to the observations. The stars selected for observations lie close to the mean loci of the colour magnitude diagram of NGC 2808. Virtually no field contamination is expected for the BHB, while some field interlopers may be present in our RHB sample. We then determined their membership from the radial velocities (also listed in Table 1), because the fairly high radial velocity of NGC 2808 $\left(+101.6 \pm 0.7 \mathrm{~km} \mathrm{~s}^{-1}\right.$, Harris 1996) ensured that very few field stars with similar radial velocities were to be expected. Thirtyseven RHB stars turned out to be cluster members based on this criterion. A close inspection revealed that most of the cluster members are grouped in a very narrow region of the colour magnitude diagram, while the field stars are typically slightly bluer and fainter than the real RHB stars. After the abundance analysis, we excluded another star (\#36742) because its $\mathrm{Fe}$ abundance is much larger than typical for stars in NGC 2808. Note that the radial velocity and the $K$ magnitude of this star only marginally agree with those of the NGC 2808 RHB members. A

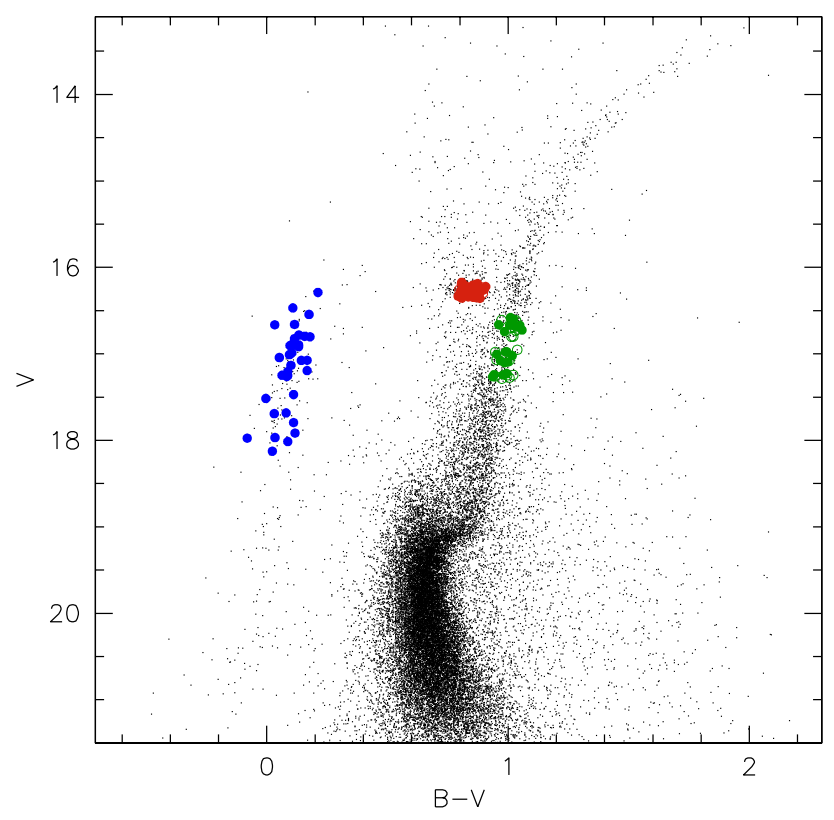

Fig. 2. Colour magnitude diagram of the inner $1.5 \leq R \leq 10.5$ region of NGC 2808. Filled circles are confirmed cluster members, while open circles (13 and 14 red $\mathrm{HB}$ and red giants respectively) are non cluster members. Different colours are used for stars on the RGB (green), RHB (red), and BHB (blue). Stars not observed are shown as dots.

similar analysis for the RGB stars indicated that 22 stars are cluster members. The average radial velocity of the 36 RHB stars is $+101.0 \pm 0.9 \mathrm{~km} \mathrm{~s}^{-1}$, in agreement with the value listed by Harris (1996). The rms scatter is $5.7 \mathrm{~km} \mathrm{~s}^{-1}$. The average radial velocity of the lower RGB (21 stars) is $+99.9 \pm 3.0 \mathrm{~km} \mathrm{~s}^{-1}$, with an rms scatter of $9.2 \mathrm{~km} \mathrm{~s}^{-1}$. The difference between the rms scatter obtained for RHB and RGB stars is not significant.

Not unexpectedly, most of the BHB stars of NGC 2808 turned out to be hotter than $11500 \mathrm{~K}$, with only six stars cooler than this limit. In the following, we will present our result for these last stars and for the RHB member stars. Results for the other stars will be presented elsewhere.

We also measured radial velocities for the BHB stars cooler than the Grundahl $u$-jump, though with fairly large errors because they are based on the O I triplet lines alone. Three of these stars are in common with the study of Recio-Blanco et al. (2004). These are $48003=2333,10449=3949$, and $37289=3841$ (the first number is our identification, the second that from Recio-Blanco et al. 2004); however, we have radial velocity only for two of them. The comparison is fairly good, with a difference of $2 \pm 3 \mathrm{~km} \mathrm{~s}^{-1}$.

Various authors found that horizontal branch stars rotate with velocities up to a few tens of $\mathrm{km} \mathrm{s}^{-1}$ for BHB stars (Peterson et al. 1995; Behr et al. 2000a,b) and slightly less for RHB ones (Carney et al. 2008). No really fast rotator was found among the 16 BHB stars observed by Recio-Blanco et al. (2004) in NGC 2808, where the maximum rotation velocity was $13 \pm$ $4 \mathrm{~km} \mathrm{~s}^{-1}$ for their star 3841, which is our star 37289 . We have checked our sample for fast rotators. To this aim, we considered the $F W H M$ of the fit to the cross-correlation peak obtained using as a template a spectrum of star of similar atmospheric parameter taken with the same instrument. To derive vsin $i$ from the FWHM the contribution caused by the instrumental profile, macro- and micro-turbulence, and thermal Doppler broadening 
Table 1. Basic data for program stars.

\begin{tabular}{|c|c|c|c|c|c|c|c|c|c|}
\hline Star & RA (J2000) & Dec (J2000) & $\begin{array}{c}B \\
(\mathrm{mag})\end{array}$ & $\begin{array}{c}V \\
(\mathrm{mag})\end{array}$ & $\begin{array}{c}K \\
(\mathrm{mag})\end{array}$ & $\begin{array}{c}V_{r} \\
\left(\mathrm{~km} \mathrm{~s}^{-1}\right)\end{array}$ & $\begin{array}{c}S / N \\
\text { HR12 }\end{array}$ & $\begin{array}{c}S / N \\
\text { HR19 }\end{array}$ & Note \\
\hline \multicolumn{10}{|c|}{ Blue HB } \\
\hline 7136 & 91149.831 & -644935.89 & 17.360 & 17.193 & 15.671 & & 7.7 & & \\
\hline 7189 & 91253.801 & -644932.72 & 16.719 & 16.544 & 16.251 & 106 & 16.0 & 11.6 & \\
\hline 8008 & 91249.328 & -644904.85 & 17.241 & 17.074 & & 117 & 9.7 & 7.5 & \\
\hline 9868 & 91201.742 & -644734.54 & 16.697 & 16.664 & & & 16.5 & 10.0 & \\
\hline 10449 & 91217.199 & -644640.87 & 16.982 & 16.803 & 15.433 & & 13.4 & & \\
\hline 10719 & 91224.790 & -644609.95 & 17.906 & 17.795 & & & 7.5 & 2.5 & \\
\hline 14516 & 91137.245 & -644727.86 & 16.775 & 16.660 & 16.073 & & 15.0 & 13.9 & \\
\hline 14598 & 91023.139 & -644715.20 & 17.000 & 16.904 & & & 12.8 & 6.5 & \\
\hline 14923 & 91127.828 & -644622.48 & 17.582 & 17.471 & & & 7.6 & 4.3 & \\
\hline 15924 & 91141.884 & -644205.67 & 17.894 & 17.975 & & & 6.2 & 4.3 & \\
\hline 31470 & 91142.487 & -645401.04 & 17.337 & 17.250 & & & 5.9 & 7.1 & \\
\hline 32324 & 91136.900 & -645305.85 & 17.219 & 17.076 & & 112 & 12.3 & 8.9 & \\
\hline 36840 & 91231.524 & -645853.27 & 18.149 & 18.126 & & & 4.8 & 1.6 & \\
\hline 37288 & 91234.354 & -645733.47 & 17.722 & 17.691 & & & 4.2 & 2.3 & \\
\hline 37289 & 91150.141 & -645733.78 & 16.954 & 16.797 & 15.427 & 95 & 9.4 & 7.9 & \\
\hline 37345 & 91212.465 & -645726.97 & 18.033 & 17.916 & & & 3.3 & 3.6 & \\
\hline 37456 & 91158.235 & -645713.01 & 17.049 & 16.917 & 16.127 & & 11.5 & 5.2 & \\
\hline 40169 & 91144.754 & -645444.00 & 17.233 & 17.133 & & & 11.8 & 7.8 & \\
\hline 40309 & 91202.205 & -645439.87 & 17.291 & 17.204 & & & 14.0 & 5.9 & \\
\hline 41077 & 91218.701 & -645417.79 & 16.939 & 16.824 & 15.946 & & 12.0 & 6.1 & \\
\hline 41388 & 91210.354 & -645410.58 & 16.993 & 16.884 & 16.192 & & 10.1 & 9.2 & \\
\hline 41586 & 91200.287 & -645406.22 & 17.013 & 16.899 & & & 12.4 & 8.9 & \\
\hline 45468 & 91147.711 & -645253.95 & 17.095 & 17.043 & & & 16.0 & 8.4 & \\
\hline 45560 & 91225.347 & -645252.13 & 17.029 & 16.897 & & & 12.7 & 11.1 & \\
\hline 46225 & 91301.673 & -645240.20 & 18.002 & 17.968 & & & 5.3 & & \\
\hline 47058 & 91223.577 & -645227.15 & 17.344 & 17.261 & & & 7.0 & & \\
\hline 48260 & 91143.336 & -645208.19 & 16.577 & 16.469 & 15.661 & & 13.6 & 11.4 & \\
\hline 48803 & 91223.235 & -645159.61 & 16.502 & 16.290 & 14.324 & 114 & 18.1 & 15.4 & \\
\hline 50078 & 91220.455 & -645140.34 & 18.101 & 18.014 & & & 3.1 & 6.9 & \\
\hline 53523 & 91144.322 & -645045.47 & 16.916 & 16.783 & 15.626 & & 11.7 & 8.9 & \\
\hline 54675 & 91146.803 & -645027.35 & 17.512 & 17.516 & & & 6.3 & 10.9 & \\
\hline 56118 & 91210.933 & -645002.59 & 17.103 & 17.009 & & & 11.2 & 12.0 & \\
\hline 56240 & 91213.574 & -645000.50 & 17.092 & 16.988 & & & 11.1 & 12.1 & \\
\hline 56998 & 91210.271 & -644947.54 & 17.311 & 17.248 & & & 11.3 & & \\
\hline 58322 & 91234.081 & -650105.81 & 17.763 & 17.683 & & & 5.1 & 1.0 & \\
\hline \multicolumn{10}{|c|}{ Red HB } \\
\hline 7858 & 91225.778 & -644911.09 & 17.192 & 16.328 & 13.988 & 95.5 & 18.0 & 21.9 & \\
\hline 8288 & 91202.282 & -644855.90 & 17.206 & 16.334 & 14.003 & 105.8 & 19.0 & 18.7 & \\
\hline 8342 & 91221.241 & -644853.89 & 17.101 & 16.298 & 14.188 & 111.2 & 20.4 & 19.6 & \\
\hline 8982 & 91248.755 & -644824.15 & 17.185 & 16.324 & 13.986 & 107.3 & 22.9 & 18.9 & \\
\hline 9792 & 91238.353 & -644738.59 & 17.217 & 16.348 & 13.900 & 105.3 & 23.4 & 17.0 & \\
\hline 10026 & 91218.448 & -644722.11 & 17.138 & 16.276 & 14.011 & 107.2 & 23.7 & & \\
\hline 10344 & 91144.067 & -644652.29 & 17.148 & 16.248 & 14.003 & 30.7 & 23.6 & 18.7 & Not member \\
\hline 10377 & 91148.520 & -644648.72 & 17.151 & 16.314 & 14.086 & 102.3 & 28.0 & 19.9 & \\
\hline 10421 & 91206.487 & -644644.20 & 17.126 & 16.247 & 13.902 & 94.2 & 23.9 & 18.4 & \\
\hline 10484 & 91200.239 & -644636.50 & 17.094 & 16.225 & 13.958 & 106.9 & 26.8 & 21.5 & \\
\hline 10574 & 91150.176 & -644626.57 & 17.082 & 16.258 & 14.237 & 55.3 & 24.7 & 21.4 & Not member \\
\hline 10769 & 91215.249 & -644603.65 & 17.112 & 16.279 & 14.109 & 99.2 & 30.6 & & \\
\hline 10878 & 91217.186 & -644547.52 & 17.193 & 16.334 & 13.967 & 98.2 & 25.4 & 17.2 & \\
\hline 11761 & 91146.147 & -644158.73 & 16.980 & 16.172 & 13.962 & 100.7 & 20.3 & 24.5 & \\
\hline 13128 & 91110.061 & -644937.54 & 17.199 & 16.345 & 14.022 & 102.0 & 24.9 & 25.8 & \\
\hline 13551 & 91140.153 & -644904.69 & 17.164 & 16.356 & 14.049 & 108.8 & 13.6 & 20.1 & \\
\hline 14280 & 91106.203 & -644753.46 & 17.117 & 16.229 & 14.157 & 11.4 & 11.1 & 20.5 & Not member \\
\hline 15084 & 91110.387 & -644551.64 & 17.073 & 16.257 & 14.024 & 100.1 & 12.3 & 19.4 & \\
\hline 15413 & 91140.070 & -644441.60 & 17.133 & 16.308 & 14.349 & 41.6 & 20.5 & 21.0 & Not member \\
\hline 15837 & 91126.451 & -644241.06 & 17.081 & 16.261 & 14.286 & 98.4 & 22.3 & 19.8 & \\
\hline 16122 & 91127.535 & -644048.86 & 17.197 & 16.302 & 14.283 & 51.5 & 13.2 & & Not member \\
\hline 29702 & 91118.545 & -645719.91 & 17.110 & 16.292 & 14.404 & 39.4 & 13.6 & 13.2 & Not member \\
\hline 29874 & 91138.076 & -645648.77 & 17.159 & 16.261 & 13.968 & 96.1 & 17.5 & 15.8 & \\
\hline 30014 & 91026.102 & -645625.55 & 17.031 & 16.212 & 14.143 & 96.1 & 13.1 & 12.0 & \\
\hline
\end{tabular}


R. G. Gratton et al.: Na-O in HB stars of NGC 2808. I.

Table 1. continued.

\begin{tabular}{|c|c|c|c|c|c|c|c|c|c|}
\hline Star & RA (J2000) & Dec (J2000) & $\begin{array}{c}B \\
(\mathrm{mag})\end{array}$ & $\begin{array}{c}V \\
(\mathrm{mag})\end{array}$ & $\begin{array}{c}K \\
(\mathrm{mag})\end{array}$ & $\begin{array}{c}V_{r} \\
\left(\mathrm{~km} \mathrm{~s}^{-1}\right) \\
\end{array}$ & $\begin{array}{c}S / N \\
\text { HR12 }\end{array}$ & $\begin{array}{c}S / N \\
\text { HR19 }\end{array}$ & Note \\
\hline 30476 & 91032.738 & -645527.53 & 17.205 & 16.341 & 14.326 & 20.9 & 14.4 & 6.7 & Not member \\
\hline 31495 & 91110.700 & -645359.04 & 17.069 & 16.265 & 14.147 & 101.2 & 23.5 & 18.3 & \\
\hline 32580 & 91110.784 & -645250.07 & 17.087 & 16.239 & 13.913 & 97.0 & 23.5 & 15.6 & \\
\hline 33521 & 91033.897 & -645156.22 & 17.100 & 16.263 & 14.207 & 47.6 & 17.5 & 12.5 & Not member \\
\hline 34706 & 91111.355 & -645049.22 & 17.148 & 16.306 & 14.081 & 96.0 & 23.4 & 20.1 & \\
\hline 34911 & 91102.365 & -645035.78 & 17.140 & 16.266 & 13.930 & 92.5 & 21.7 & 14.6 & \\
\hline 34953 & 91139.265 & -645033.62 & 17.222 & 16.341 & 13.340 & 117.3 & 22.0 & 17.2 & \\
\hline 35212 & 91113.105 & -645016.45 & 17.120 & 16.315 & 14.181 & 100.1 & 17.5 & 28.3 & \\
\hline 35290 & 91105.628 & -645009.90 & 17.241 & 16.358 & 14.052 & 97.6 & 14.7 & & \\
\hline 36347 & 91103.699 & -650147.67 & 17.069 & 16.234 & 14.407 & 28.9 & 17.2 & 13.3 & Not member \\
\hline 36638 & 91227.005 & -645942.31 & 16.996 & 16.189 & 14.091 & 97.3 & 21.8 & 17.0 & \\
\hline 36731 & 91148.708 & -645921.02 & 17.060 & 16.204 & 14.045 & 104.0 & 23.8 & 15.6 & \\
\hline 36742 & 91155.924 & -645918.54 & 17.101 & 16.290 & 14.462 & 118.5 & 21.5 & 13.0 & Not member \\
\hline 37086 & 91147.948 & -645804.76 & 17.055 & 16.257 & 14.000 & -5.0 & 19.8 & 18.4 & Not member \\
\hline 37747 & 91148.167 & -645645.31 & 17.104 & 16.289 & 13.990 & 94.7 & 19.5 & 11.5 & \\
\hline 39743 & 91158.834 & -645457.92 & 17.162 & 16.302 & 13.994 & 105.5 & 22.1 & 17.8 & \\
\hline 41995 & 91247.108 & -645356.37 & 17.126 & 16.333 & 13.566 & 96.3 & 21.8 & 17.4 & \\
\hline 49327 & 91252.349 & -645151.06 & 17.085 & 16.245 & 13.977 & 104.6 & 23.7 & 16.9 & \\
\hline 52411 & 91251.438 & -645102.46 & 17.060 & 16.187 & 13.795 & 97.5 & 20.1 & 18.7 & \\
\hline 56969 & 91230.387 & -644947.78 & 17.175 & 16.340 & 14.063 & 92.9 & 21.5 & 19.8 & \\
\hline 57999 & 91149.570 & -650329.51 & 17.162 & 16.280 & 14.097 & 16.0 & 15.9 & 4.0 & Not member \\
\hline 58106 & 91143.156 & -650240.83 & 17.030 & 16.209 & 14.082 & 108.8 & 18.7 & 14.1 & \\
\hline 59879 & 91321.297 & -645653.00 & 17.089 & 16.229 & 13.928 & 96.5 & 16.2 & 17.4 & \\
\hline 60283 & 91321.880 & -645603.05 & 17.128 & 16.221 & 13.966 & 101.1 & 18.4 & 21.7 & \\
\hline 62438 & 91356.774 & -645139.58 & 17.090 & 16.217 & 14.192 & 31.5 & 10.7 & 19.3 & Not member \\
\hline \multicolumn{10}{|c|}{ Lower RGB } \\
\hline 111 & 91343.797 & -644911.96 & 17.959 & 17.006 & 14.552 & 80.9 & 10.5 & 16.3 & \\
\hline 273 & 91340.612 & -644847.20 & 18.083 & 17.077 & 14.757 & 3.1 & 5.6 & 12.9 & Not member \\
\hline 9505 & 91248.373 & -644756.23 & 18.037 & 17.020 & 14.318 & 107.1 & 11.5 & 16.2 & \\
\hline 10162 & 91245.713 & -644709.20 & 17.782 & 16.724 & 14.049 & 105.7 & 18.9 & & \\
\hline 10273 & 91225.930 & -644659.52 & 18.221 & 17.227 & 14.465 & 113.5 & 14.3 & 12.1 & \\
\hline 11925 & 91222.769 & -644106.29 & 17.814 & 16.800 & 14.209 & 198.3 & 15.9 & & Not member \\
\hline 14174 & 91048.268 & -644806.23 & 17.967 & 16.978 & 14.435 & 104.8 & 12.0 & & \\
\hline 14713 & 91105.928 & -644656.55 & 17.727 & 16.742 & 14.067 & 107.0 & 15.6 & & \\
\hline 23937 & 91017.427 & -645227.52 & 18.206 & 17.259 & 14.712 & 10.3 & 9.3 & 8.5 & Not member \\
\hline 29619 & 91140.275 & -645742.00 & 17.821 & 16.799 & 14.420 & 14.1 & 10.7 & 12.1 & Not member \\
\hline 30526 & 91053.972 & -645522.66 & 18.020 & 17.068 & 14.747 & 14.3 & 7.2 & 8.0 & Not member \\
\hline 30550 & 91125.095 & -645520.18 & 17.723 & 16.690 & 14.058 & 102.9 & 10.3 & & \\
\hline 30740 & 91116.898 & -645459.59 & 18.182 & 17.240 & 14.878 & 101.2 & 7.3 & 10.9 & \\
\hline 33684 & 91059.201 & -645148.84 & 18.094 & 17.095 & 14.588 & 110.8 & 13.4 & & \\
\hline 34075 & 91132.786 & -645127.40 & 17.991 & 16.953 & 14.274 & 52.6 & 14.2 & & Not member \\
\hline 36283 & 91117.389 & -650215.93 & 18.267 & 17.245 & 15.000 & 57.2 & 9.6 & 9.6 & Not member \\
\hline 36517 & 91107.907 & -650031.64 & 17.581 & 16.608 & 14.277 & -3.4 & 14.2 & 7.8 & Not member \\
\hline 36833 & 91221.447 & -645856.24 & 17.925 & 16.978 & 14.860 & 53.0 & 6.7 & 10.6 & Not member \\
\hline 36928 & 91238.652 & -645836.76 & 18.281 & 17.275 & 14.771 & -25.9 & 10.9 & & Not member \\
\hline 37188 & 91303.884 & -645748.84 & 18.243 & 17.247 & 14.795 & 46.0 & 10.2 & 6.7 & Not member \\
\hline 37743 & 91215.973 & -645645.45 & 18.087 & 17.102 & 14.509 & 95.3 & 10.6 & & \\
\hline 38530 & 91211.733 & -645550.51 & 17.625 & 16.602 & 13.866 & 88.2 & 15.6 & & \\
\hline 39189 & 91301.121 & -645517.94 & 17.623 & 16.662 & 14.051 & 100.9 & 17.4 & 16.0 & \\
\hline 40873 & 91244.562 & -645423.35 & 17.675 & 16.669 & 13.961 & 91.1 & 17.2 & & \\
\hline 42578 & 91237.677 & -645345.07 & 18.261 & 17.287 & 14.999 & -6.3 & 11.0 & 10.5 & Not member \\
\hline 44947 & 91217.666 & -645302.46 & 18.200 & 17.264 & & 92.5 & 12.7 & & \\
\hline 48667 & 91251.544 & -645201.25 & 17.761 & 16.730 & 14.145 & 50.0 & 16.4 & 16.8 & Not member \\
\hline 50027 & 91224.989 & -645141.03 & 18.223 & 17.244 & 14.799 & 108.9 & 12.1 & 11.6 & \\
\hline 51996 & 91222.779 & -645109.59 & 17.972 & 16.978 & 14.450 & 102.1 & 12.9 & 14.7 & \\
\hline 55130 & 91238.880 & -645019.34 & 17.668 & 16.648 & 14.008 & 88.3 & 13.8 & & \\
\hline 57250 & 91156.043 & -644943.17 & 17.590 & 16.580 & 13.829 & 110.8 & 18.6 & & \\
\hline 58411 & 91209.748 & -650029.04 & 18.045 & 17.077 & 14.760 & 90.0 & 8.4 & 9.0 & \\
\hline 58515 & 91215.966 & -650000.61 & 17.968 & 16.975 & 14.482 & 89.7 & 10.9 & & \\
\hline 60828 & 91334.205 & -645456.58 & 17.646 & 16.613 & 14.211 & 25.1 & 9.7 & 12.0 & Not member \\
\hline 63132 & 91309.746 & -645015.15 & 17.719 & 16.670 & 14.019 & 105.8 & 14.6 & 25.1 & \\
\hline
\end{tabular}


should be taken into account, both for the stars and the template, and the relation should be calibrated (see Lucatello \& Gratton 2003). However, the FWHM of the cross correlation of spectra of stars with similar atmospheric parameters with the same template gives a qualitative indication of the rotational rate. To this aim we used the spectra taken with grating HR12, because in HR19 the number of stellar lines is much smaller and the spectra are dominated by telluric lines.

Among red HB stars we found no evidence of a considerable variation in the measured $F W H M$ s, which range from $\sim 28$ to $\sim 40 \mathrm{~km} \mathrm{~s}^{-1}$. While these values cannot exclude the presence of stars rotating as fast as $v \sin i \sim 5-7 \mathrm{~km} \mathrm{~s}^{-1}$ (typical values for field RHB stars observed by Carney et al. 2008), just the accounting of the instrumental profile of template and object stars at the spectral resolution used $(R \simeq 18700$ ) leaves no room for high rotational velocities. We cannot draw any conclusions concerning blue HB stars: the paucity of lines combined with the quite low signal-to-noise ratio makes the estimates of $F W H M$ fairly uncertain as well as highly affected by noise, residuals of telluric subtraction, etc.

\section{Analysis}

\subsection{Atmospheric parameters}

The programme stars cover a wide range in effective temperature. It is therefore difficult to mantain a uniform criterium to derive atmospheric parameters. In addition, the low $S / N$ and the limited spectral coverage of the spectra prevent a full spectroscopic analysis. The adopted procedure was the following.

For the RHB and RGB stars, effective temperatures were derived from the $B-V$ and $V-K$ colours, using the calibration of Alonso et al. (1999, with the erratum of Alonso et al. 2001). The colours were dereddened using the $E(B-V)$ values from the updated on-line version of the Harris (1996) catalogue and the $E(V-K) / E(B-V)$ value from Cardelli et al. (1989). The calibrations require input values for the metallicity $[\mathrm{A} / \mathrm{H}]$. We adopted the value obtained by Carretta et al. (2006). We assign weight 4 to the $B-V$ colours, and 1 to the $V-K$ ones, because the programme stars are very faint for the 2MASS observations. For the blue HB stars we used the $(B-V)-T_{\text {eff }}$ calibration by Kurucz $^{4}$.

Errors are mainly caused by uncertainties in the colours. For the RHB stars, an error of $0.01 \mathrm{mag}$ in $B-V$ causes an error of $25 \mathrm{~K}$ in the effective temperature; a slightly smaller error of $20 \mathrm{~K}$ is obtained for red giants. A similar photometric error for the BHB stars causes an error of $\sim 300 \mathrm{~K}$. We assumed errors of 50 and $300 \mathrm{~K}$ as representative values for the internal errors in the temperatures. Systematic errors caused by scale errors or incorrect parameters for the cluster are likely larger. However, they are not really important in our discussion.

The surface gravities were obtained from the masses, luminosities, and effective temperatures. For the masses we adopted values of $0.9,0.7$, and $0.5 M_{\odot}$ for stars on the RGB, RHB, and BHB (see Gratton et al. 2010, for a discussion of adequate values for the different sequences). Note that these values are not very critical, because a variation of $0.1 M_{\odot}$ changes gravities by 0.07 and 0.10 dex for RHB and BHB stars. The effects on $[\mathrm{Na} / \mathrm{Fe}]$ are of 0.11 and $0.08 \mathrm{dex}$, and those on $[\mathrm{O} / \mathrm{Fe}] 0.02$ and 0.13 dex, for BHB and RHB respectively. The bolometric corrections needed in these derivations were obtained using calibrations consistent with those used for the effective temperatures

\footnotetext{
${ }^{4}$ See kurucz.harvard.edu.
}

(Alonso et al. 1999, for the red giant and RHB stars, and Kurucz for the BHB stars). The distance moduli used were taken from the Harris catalogue.

Errors in gravities are very small. The assumption about masses is likely correct within 10\% (0.04 dex error in the gravities), while that on the effective temperature and luminosity causes errors in gravities not larger than $\sim 2 \%$ for the RHB stars and red giants, and $\sim 12 \%$ for the BHB stars. The error in gravities is then not larger than 0.05 dex for the cool stars, while it may be 0.10 dex for the warm ones.

The same metal abundance of $[\mathrm{A} / \mathrm{H}]=-1.14$ and microturbulence velocity of $1.8 \mathrm{~km} \mathrm{~s}^{-1}$ were adopted for all RHB stars. The metal abundance is the average value determined in Carretta et al. (2006), and agrees well with the average $[\mathrm{Fe} / \mathrm{H}]=-1.18 \pm$ 0.01 (rms of $0.07 \mathrm{dex}$ ) we determined from the 36 RHB stars. The adopted microturbulence velocity sets to zero the average of the trends of $\mathrm{Fe}$ abundances with expected line strength (see Magain 1984, for a justification of this approach). We prefer to adopt this average value rather than values appropriate for each star because we were able to measure equivalent widths $(E W)$ only for a limited number of Fe lines (in the range 6-16) for each star, with fairly large errors $(\sim 13 \mathrm{~m} \AA$, evaluated from the rms of measures for the same line across the RHB sample), and over a limited range of $E W \mathrm{~s}$. The uncertainties in the individual slopes, which agree with the observed rms scatter, would translate into a large error bar of $\pm 0.5 \mathrm{~km} \mathrm{~s}^{-1}$. This is likely more than the expected star-to-star scatter, which we estimated to be $\sim 0.3 \mathrm{~km} \mathrm{~s}^{-1}$ in analogy with the RGB stars studied by Carretta et al. (2009a). We will adopt this last value as the error bar of our microturbulent velocities. We were unable to derive microturbulent velocities for BHB stars because too few lines were measured. The value we adopted $\left(2 \mathrm{~km} \mathrm{~s}^{-1}\right)$ is in the middle of the range usually found in previous analysis of BHB stars (Lambert et al. 1992; Behr et al. 1999, 2000b; Kinman et al. 2000; Fabbian et al. 2005; Villanova et al. 2009; Marino et al. 2011).

While a single Fe II line was measured in our spectra (at $5991.38 \AA$ A), abundances derived from this line agree well with those obtained from the Fe I lines: on average we obtained $[\mathrm{Fe} \mathrm{II} / \mathrm{H}]=-1.13 \pm 0.03$. This supports the choice of the atmospheric parameters adopted throughout this paper.

Table 2 lists the effective temperatures $T_{\text {eff }}$ and surface gravities $\log g$ we used in the analysis of the programme stars, as well as the abundances we obtained from our analysis for lines of Fe I, Fe II, O I, Na I, Mg I, Si I, Ca I, and Ba II. Abundances were estimated from equivalent widths. The oscillator strengths for Fe lines were generally obtained from the VALD database (Kupka et al. 2000). The same source was used for the $\mathrm{Mg}$ line at $8213.04 \AA$, for four lines of $\mathrm{Si}(5948.55,7918.38,7932.35$ and $7944.00 \AA$ ), for the Ca line at $5857.46 \AA$, and for the Ba II line at $5853.69 \AA$. Note that not all stars were observed with both gratings. As a consequence, we were not able to measure the abundances of all elements in each star. For this reason we have no O abundance for star \#10449, for instance.

For O I and $\mathrm{Na}$ I lines, the abundances include corrections due to departures from LTE, which are large for the transition and stars of interest here. The non-LTE corrections used are estimated from standard statistical equilibrium calculations. They include integration of the radiation transfer equation throughout the stellar atmosphere, with corrections to the populations of the individual levels and to the source function computed at all optical depth. There are uncertainties, related to the approximation present and to the poor knowledge of the relevant cross sections. In particular the treatment of collisions with $\mathrm{H} \mathrm{I}$ atoms 
R. G. Gratton et al.: Na-O in HB stars of NGC 2808. I.

Table 2. Atmospheric parameters and abundances.

\begin{tabular}{|c|c|c|c|c|c|c|c|c|c|c|c|c|c|c|c|c|}
\hline \multirow[t]{2}{*}{ Star } & \multirow{2}{*}{$\begin{array}{l}T_{\text {eff }} \\
(\mathrm{K})\end{array}$} & \multirow{2}{*}{$\begin{array}{l}\log g \\
(\operatorname{dex})\end{array}$} & \multirow{2}{*}{\multicolumn{2}{|c|}{$\begin{array}{c}{[\mathrm{Fe} / \mathrm{H}]_{\mathrm{I}}} \\
\langle\rangle\end{array}$}} & \multirow[b]{2}{*}{$\mathrm{rms}$} & \multirow[t]{2}{*}[\mathrm{Fe}/\mathrm{H}]{$_{\mathrm{II}}$} & \multicolumn{3}{|c|}{$[\mathrm{O} / \mathrm{Fe}]$} & \multicolumn{3}{|c|}{$[\mathrm{Na} / \mathrm{Fe}]$} & \multirow[t]{2}{*}[\mathrm{Mg}/\mathrm{Fe}]{} & \multirow[t]{2}{*}[\mathrm{Si}/\mathrm{Fe}]{} & \multirow[t]{2}{*}[\mathrm{Ca}/\mathrm{Fe}]{} & \multirow[t]{2}{*}[\mathrm{Ba}/\mathrm{Fe}]{} \\
\hline & & & & & & & lines & \langle\rangle & $\mathrm{rms}$ & lines & \langle\rangle & rms & & & & \\
\hline & & & & & & & & \multicolumn{2}{|c|}{ Blue HB } & & & & & & & \\
\hline 7189 & 10020 & 3.30 & & & & & 3 & \multicolumn{2}{|c|}{-0.23} & 2 & 0.44 & 0.07 & & & & \\
\hline 8008 & 10270 & 3.33 & & & & & 2 & \multicolumn{2}{|l|}{-0.29} & 1 & 0.45 & & & & & \\
\hline 10449 & 9900 & 3.29 & & & & & & & & 1 & 0.25 & & & & & \\
\hline 32324 & 11120 & 3.37 & & & & & 2 & \multicolumn{2}{|l|}{-0.66} & 2 & 0.61 & 0.07 & & & & \\
\hline 37289 & 10560 & 3.29 & & & & & 1 & \multicolumn{2}{|l|}{-0.71} & 2 & 0.61 & 0.09 & & & & \\
\hline 48803 & 9060 & 3.17 & & & & & 3 & \multicolumn{2}{|l|}{-0.75} & 2 & 0.65 & 0.01 & & & & \\
\hline & & & & & & & & Red H & & & & & & & & \\
\hline 7858 & 5490 & 2.53 & 14 & -1.27 & 0.29 & -1.09 & 2 & 0.54 & 0.03 & 4 & -0.15 & 0.17 & 0.16 & 0.04 & 0.61 & 0.49 \\
\hline 8288 & 5470 & 2.52 & 15 & -1.25 & 0.32 & -0.89 & 3 & 0.60 & 0.09 & 4 & 0.09 & 0.08 & 0.12 & 0.30 & 0.20 & 0.65 \\
\hline 8342 & 5680 & 2.58 & 15 & -1.11 & 0.28 & -1.07 & 2 & 0.43 & 0.04 & 4 & 0.28 & 0.20 & 0.36 & 35 & 45 & 0.52 \\
\hline 8982 & 5490 & 2.53 & 16 & -1.20 & 0.30 & -1.20 & 1 & 0.38 & & 4 & -0.02 & 0.07 & 0.26 & 0.17 & 0. & 0.19 \\
\hline 9792 & 5450 & 2.52 & 16 & -1.26 & 0.30 & -1.26 & 2 & 0.79 & 0.21 & 4 & 0.06 & 0.07 & 0.04 & 0.47 & 0.41 & 0.42 \\
\hline 10026 & 5510 & 2.51 & 6 & -1.16 & 0.18 & -1.32 & & & & 2 & 0.00 & 0.17 & & & 0.45 & 0.56 \\
\hline 10377 & 5570 & 2.55 & 16 & -1.19 & 0.19 & -1.35 & 3 & 0.67 & 0.26 & 4 & -0.04 & 0.27 & 0.09 & 0.60 & 0.15 & 0.00 \\
\hline 10421 & 5460 & 2.48 & 16 & -1.19 & 0.21 & -1.26 & 3 & 0.15 & 0.02 & 4 & -0.17 & 0.37 & 0.19 & 0.49 & 0.42 & \\
\hline 10484 & 5500 & 2.49 & 16 & -1.13 & 0.16 & -1.03 & 2 & 0.54 & 0.02 & 4 & -0.21 & 0.21 & 0.07 & 0.61 & 0.54 & 0.18 \\
\hline 10769 & 5600 & 2.55 & 6 & -1.05 & 0.07 & -1.08 & & & & 2 & 0.00 & 0.02 & & & 0.49 & 0.18 \\
\hline 10878 & 5490 & 2.53 & 16 & -1.16 & 0.19 & -0.82 & 2 & 0.49 & 0.20 & 4 & 0.07 & 0.34 & 0.09 & 0.53 & 0.41 & -0.03 \\
\hline 11761 & 5640 & 2.52 & 14 & -1.12 & 0.28 & -1.29 & 3 & 0.44 & 0.16 & 3 & 0.23 & 0.30 & 0.28 & 0.32 & & 0.15 \\
\hline 13128 & 5510 & 2.54 & 13 & -1.22 & 0.20 & -1.17 & 2 & 0.18 & 0.01 & 4 & -0.18 & 0.20 & 0.11 & 0.14 & 0.35 & -0.22 \\
\hline 13551 & 5610 & 2.58 & 13 & -1.26 & 0.22 & -0.83 & 3 & 0.51 & 0.14 & 4 & 0.02 & 0.21 & 0.21 & 0.29 & 0.48 & 0.45 \\
\hline 15084 & 5620 & 2.55 & 16 & -1.12 & 0.24 & -1.32 & 3 & 0.47 & 0.01 & 4 & 0.11 & 0.20 & 0.35 & 0.47 & 0.63 & 0.42 \\
\hline 15837 & 5690 & 2.57 & 15 & -1.06 & 0.22 & -0.73 & 2 & 0.33 & 0.14 & 4 & 0.08 & 0.3 & & 0. & 0.44 & 0.12 \\
\hline 29874 & 5450 & 2.48 & 14 & -1.04 & 0.22 & -1.09 & 3 & 0.64 & 0.10 & 4 & -0.09 & & & 0. & 0. & 0.43 \\
\hline 30014 & 5660 & 2.54 & 14 & -1.12 & 0.22 & -1.06 & 3 & 0.40 & 0.13 & 4 & 0.33 & 0.33 & 0.1 & 0. & 0.92 & 0.29 \\
\hline 31495 & 5680 & 2.57 & 14 & -1.17 & 0.27 & -1.03 & 3 & 0.39 & 0.29 & 4 & 0.34 & 0.37 & 0.33 & 0.04 & 0.47 & 0.49 \\
\hline 32580 & 5520 & 2.50 & 14 & -1.18 & 0.20 & -1.18 & 2 & 0.38 & 0.19 & 4 & -0.06 & 0.33 & 0.27 & 0.38 & 0.38 & -0.19 \\
\hline 34706 & 5560 & 2.55 & 15 & -1.23 & 0.17 & -1.12 & 3 & 0.54 & 0.09 & 4 & -0.08 & 0.27 & 0.10 & 0.32 & 0.59 & 0.31 \\
\hline 34911 & 5470 & 2.49 & 13 & -1.16 & 0.24 & -1.30 & 2 & 0.35 & 0.37 & 4 & -0.20 & 0.25 & 0.01 & 0.64 & 0.31 & 0.29 \\
\hline 34953 & 5460 & 2.52 & 13 & -1.27 & 0.26 & -1.58 & 1 & 0.69 & & 4 & -0.34 & 0.29 & 0.01 & 0.10 & 0.65 & 0.41 \\
\hline 35212 & 5670 & 2.59 & 12 & -1.31 & 0.32 & -1.24 & 3 & 0.14 & 0.05 & 4 & 0.28 & 0.28 & & 0.17 & 0.33 & 0.18 \\
\hline 35290 & 5460 & 2.53 & 6 & -1.29 & 0.17 & -1.02 & & & & 2 & -0.04 & 0.07 & & & 0.10 & -0.11 \\
\hline 36638 & 5680 & 2.54 & 14 & -1.06 & 0.27 & -1.06 & 2 & 0.40 & 0.23 & 4 & 0.14 & 0.35 & 0.47 & 0.47 & 0.49 & 0.74 \\
\hline 36731 & 5550 & 2.50 & 15 & -1.15 & 0.36 & -1.35 & 3 & 0.51 & 0.08 & 3 & -0.12 & 0.13 & 0.28 & 0.37 & 0.45 & 0.59 \\
\hline 37747 & 5600 & 2.55 & 16 & -1.11 & 0.37 & -1.16 & 2 & 0.32 & 0.01 & 4 & 0.20 & 0.29 & 0.13 & 0.50 & 0.25 & 0.22 \\
\hline 39743 & 5500 & 2.52 & 14 & -1.18 & 0.19 & -1.18 & 3 & 0.32 & 0.36 & 4 & -0.01 & 0.13 & 0.20 & 0.58 & 0.54 & 0.01 \\
\hline 41995 & 5690 & 2.60 & 14 & -1.20 & 0.23 & -1.16 & 3 & 0.37 & 0.26 & 4 & -0.02 & 0.19 & 0.38 & 0.34 & 0.07 & 0.07 \\
\hline 49327 & 5560 & 2.52 & 14 & -1.24 & 0.26 & -0.95 & 3 & 0.56 & 0.23 & 4 & -0.26 & 0.15 & 0.14 & 0.45 & 0.49 & -0.13 \\
\hline 52411 & 5460 & 2.46 & 15 & -1.19 & 0.21 & -1.31 & 3 & 0.45 & 0.15 & 4 & 0.22 & 0.22 & 0.37 & 0.34 & 0.32 & 0.47 \\
\hline 56969 & 5560 & 2.56 & 15 & -1.23 & 0.22 & -1.21 & 3 & 0.46 & 0.06 & 4 & -0.10 & 0.19 & 0.34 & 0.46 & 0.54 & 0.46 \\
\hline 58106 & 5640 & 2.53 & 11 & -1.13 & 0.16 & -0.96 & 1 & 0.13 & & 4 & 0.29 & 0.06 & 0.22 & 0.42 & 0.41 & 0.28 \\
\hline 59879 & 5510 & 2.49 & 12 & -1.30 & 0.24 & -1.09 & 3 & 0.65 & 0.14 & 4 & -0.13 & 0.20 & 0.14 & & 0.41 & -0.09 \\
\hline 60283 & 5430 & 2.46 & 15 & -1.11 & 0.22 & -1.00 & 3 & 0.38 & 0.11 & 4 & -0.05 & 0.21 & 0.53 & 0.27 & 0.41 & -0.21 \\
\hline
\end{tabular}

is critical and poorly known. Because different recipes are used, various non-LTE estimates produce somewhat different results, although there is generally quite a good consensus on the sign and order of magnitude of the corrections, in particular for quite simple atoms like $\mathrm{O}$ I and $\mathrm{Na}$ I. For the RHB stars, we used the corrections listed by Gratton et al. (1999), whose cross sections for collisions with $\mathrm{H} \mathrm{I}$ atoms were tuned to reproduce observations of RR Lyrae stars. The corrections are $\sim 0.5$ dex for O I and $\sim 0.2$ dex for $\mathrm{Na}$, in both cases decreasing the LTE abundances. Very recently, new Na non-LTE corrections were published by Lind et al. (2011), making use of a more extended model atom and moreover updated data about cross sections. For the lines used and the combination of atomic parameters appropriate for the programme stars, these corrections are about 0.3 dex larger than those adopted here and this difference is quite uniform. Were we to adopt these corrections, the $\mathrm{Na}$ abundances would be about 0.3 dex smaller. However, we prefer to keep here the older correction by Gratton et al. (1999) for uniformity with the red giants studied by Carretta et al. (2006). However, even these corrections were not computed for stars as hot as our BHB stars. In this case, we used the results of the statistical equilibrium calculations by Takeda (1997) for O I (with corrections to $\mathrm{O}$ abundances in the range $1-1.5 \mathrm{dex}$, and by Mashonkina et al. (2000) for $\mathrm{Na}$ I (in this case the corrections are $\sim 0.5 \mathrm{dex}$ ). We alert the reader that there might be some offsets between these different sets of corrections. These offsets are difficult to quantify accurately. If we compare non-LTE abundance corrections for O triplet lines by Takeda (1997) with those by Gratton et al. (1999) for stars with $T_{\text {eff }}=7000 \mathrm{~K}$, however, where both are available, we find that the first ones are smaller by $\sim 0.1 \mathrm{dex}$ ( $\sim 0.5$ vs. $\sim 0.6$ dex). A similar comparison for the Na non-LTE corrections of Mashonkina et al. (2000) shows that these are smaller by $\sim 0.2$ dex than those by Gratton et al. (1999) $(\sim 0.4$ vs. $\sim 0.6 \mathrm{dex})$. We conclude that uncertainties in the non-LTE corrections may be likely as large as a few tenths of a dex (see also comparisons in Lind et al. 2011, which are appropriate to 
cooler stars, however). Hence, while these uncertainties are not negligible, it is difficult to conceive that they may cause the main results of this paper.

The doublet of $\mathrm{Al} \mathrm{I}$ at 7835-36 $\AA$ is actually in the observed spectral range. We carefully examined the relevant region in the spectra of all RHB stars, but we did not find any evidence for it. The line is also not detectable in the spectrum we obtained by summing all $36 \mathrm{HB}$ stars. From this last spectrum (which has a $S / N>100$ at the relevant wavelength) we derived an upper limit of $10 \mathrm{~m} \AA$ to the equivalent width for each component of the doublet. This yields an upper limit of $[\mathrm{Al} / \mathrm{Fe}]<0.15$, which is similar to the $\mathrm{Al}$ abundances obtained for the most Al-poor RGB stars in NGC 2808 (Carretta et al. 2009b). No Al detection is possible in the low $S / N$ spectra of BHB stars.

\subsection{Error analysis}

Error analysis was made in the usual way, by repeating the abundance derivation by modifying a single parameter each time. Relevant data are given in Table 3. The last column gives an estimate of the total internal errors, estimated using the sensitivities listed above, as well as the errors in the individual parameters given in lines 2 and 3 for blue and red HB stars, respectively. In general, the main source of uncertainty is the measure of the equivalent widths, which is not surprising considering the fairly low $S / N$ of our spectra. In a few cases $(\mathrm{Fe}, \mathrm{O}$, and $\mathrm{Na}$ ) a significant contribution comes from errors in the effective temperatures and in the microturbulent velocities.

For most elements (Fe, $\mathrm{Mg}, \mathrm{Si}, \mathrm{Ca}$, and $\mathrm{Ba}$ ) the dispersion of individual values (for RHB stars alone) agrees fairly well with these estimates of the internal errors. The case of $\mathrm{Na}$ and $\mathrm{O}$ will be discussed in the next section.

\section{The $\mathrm{Na}-\mathrm{O}$ anticorrelation along the $\mathrm{HB}$ of NGC 2808}

Figure 3 shows the Na-O anticorrelation we obtained for the HB stars of NGC 2808. Different symbols are used for blue and red HB stars. For comparison we also plotted the Na-O anticorrelation for red giants by Carretta et al. (2006). We recall that the extreme blue HB stars, hotter than $11500 \mathrm{~K}$, are not considered here, because their surface abundance is not related in a simple way to their original composition. The moderately BHB stars for which we may provide sensible $\mathrm{Na}$ and $\mathrm{O}$ abundances are all $\mathrm{O}$-poor and $\mathrm{Na}$-rich, with average values of $[\mathrm{O} / \mathrm{Fe}]=-0.59 \pm 0.12$ and $[\mathrm{Na} / \mathrm{Fe}]=+0.44 \pm 0.06$. The rms scatter of individual values of 0.24 dex and 0.15 dex agree with the internal errors of Table 3 , so that we cannot conclude about any internal scatter in this small sample of six stars, even if there is a hint that the stars might be distributed into two groups. We also notice that systematic errors caused by, e.g. the corrections for non-LTE effects (which are rather large for the $\mathrm{O}$ lines) are certainly much larger than the error bars given above for the average abundances, which stems from the dispersion of individual values alone. However, the comparison of the $\mathrm{Na}$ and $\mathrm{O}$ abundances for these stars with those obtained for red giants suggests that they descend from the moderately O-poor red giants.

On the other hand, all RHB stars are much richer in $\mathrm{O}$ than the BHB stars, with positive values of $[\mathrm{O} / \mathrm{Fe}]$. These stars span a fairly large range of $\mathrm{Na}$ abundances, and the rms scatter of $[\mathrm{Na} / \mathrm{Fe}]$ values of $0.18 \pm 0.03$ dex appears to be significantly larger than the observational error of $0.11 \mathrm{dex}$. While not extremely significant, there is a clear hint for a $\mathrm{Na}-\mathrm{O}$
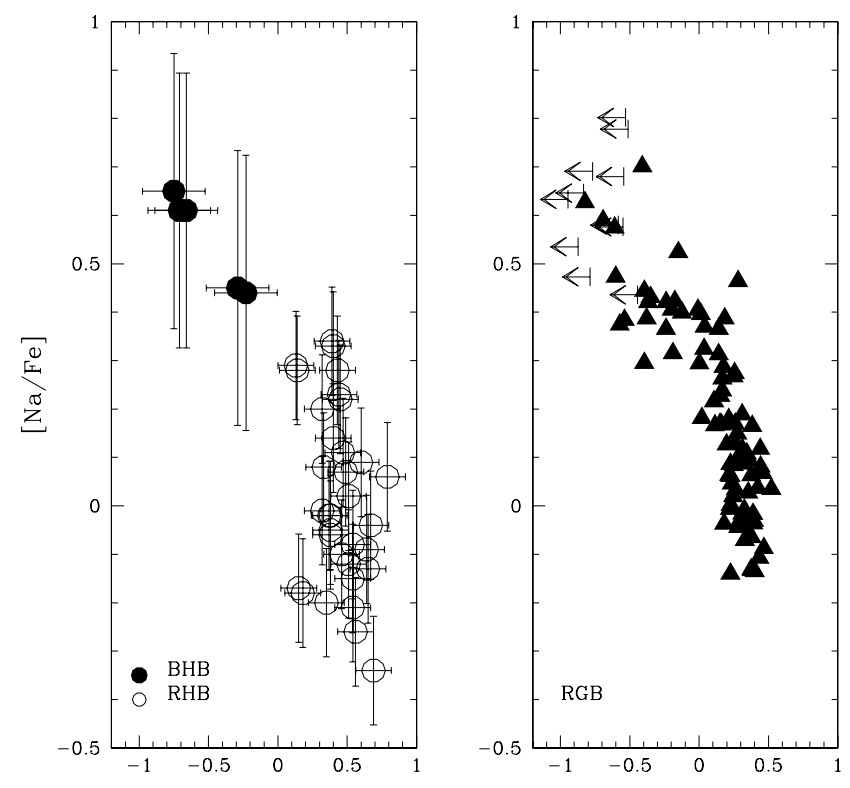

$[\mathrm{O} / \mathrm{Fe}]$

Fig. 3. Left panel: Na-O anticorrelation for HB stars of NGC 2808 Filled circles are BHB stars; open circles are RHB stars. Right panel: the same, but for red giants (from Carretta et al. 2006).

anticorrelation. The correlation coefficient is $r=-0.34$ over 31 stars, which has less than $5 \%$ chance of being a random effect. In addition, we notice that there is a very significant correlation between the $\mathrm{Na}$ abundances obtained from the resonance D-doublet and the subordinate one at 8183-94 $\AA$. This suggests that the red $\mathrm{HB}$ stars have not a single value of $\mathrm{Na}$ and $\mathrm{O}$ abundances, with only observational scatter; instead they seem to include at least two different populations. This means that only a fraction of the RHB stars of NGC 2808 descends from the primordial population (see Carretta et al. 2010, for a definition of primordial population). The comparison with red giants shows that there is a very good correspondence between the RHB stars and the O-rich sequence in NGC 2808; we incidentally note here that also O-rich red giants display a distinct spread in Na abundances, so that many of them are classified as belonging to the intermediate population according to the definition by Carretta et al. (2009a).

While we cannot determine directly the He abundances for the various populations on the HB of NGC 2808, we may propose values that are compatible with their colours and magnitudes. This matter has been considered in depth in previous investigations. For instance, D'Antona et al. (2005) considered three populations with $Y=0.24, Y=0.26-0.29$, and $Y=0.40$, with respectively 50,30 , and $20 \%$ of the stars, and found that this mix is able to reproduce both the main sequence and the HB of NGC 2808. The first group corresponds to the RHB, the second to the BHB, and the third to extreme BHB (not sampled in this paper). The same proportions are found for $\mathrm{P}, \mathrm{I}$, and $\mathrm{E}$ stars along the RGB by Carretta et al. (2010). This suggests the identification of the He-poor stars and RHB with the P-stars, of the He-intermediate and BHB stars with the I-stars, and of the He-rich and extreme BHB ones with the E-stars. As expected from this scenario, we indeed found a clear correlation between the $\mathrm{Na} / \mathrm{O}$ abundance ratio and colour and temperature along the HB in NGC 2808 (see Fig. 4). This is obvious when comparing the O-poor, Na-rich BHB with the O-rich RHB stars. While 
Table 3. Sensitivity and error analysis.

\begin{tabular}{lcrrccc}
\hline \hline Parameter & $T_{\text {eff }}$ & $\log g$ & {$[\mathrm{~A} / \mathrm{H}]$} & $v_{t}$ & $E W$ & Total \\
\hline Variation & $+100 \mathrm{~K}$ & $+0.5 \mathrm{dex}$ & $+0.1 \mathrm{dex}$ & $+0.5 \mathrm{~km} \mathrm{~s}^{-1}$ & $+10 \mathrm{~m} \AA$ & \\
Error BHB & $300 \mathrm{~K}$ & $0.1 \mathrm{dex}$ & $0.1 \mathrm{dex}$ & $0.5 \mathrm{~km} \mathrm{~s}^{-1}$ & $20 \mathrm{~m} \AA$ & \\
Error RHB & $50 \mathrm{~K}$ & $0.1 \mathrm{dex}$ & $0.1 \mathrm{dex}$ & $0.3 \mathrm{~km} \mathrm{~s}^{-1}$ & $13 \mathrm{~m} \AA$ & \\
\hline & & & Blue HB & & & \\
{$[\mathrm{O} / \mathrm{Fe}]$} & 0.025 & 0.016 & 0.008 & -0.129 & 0.085 & 0.226 \\
{$[\mathrm{Na} / \mathrm{Fe}]$} & 0.060 & -0.165 & -0.003 & -0.014 & 0.109 & 0.284 \\
\hline & & & Red HB & & & \\
{$[\mathrm{Fe} / \mathrm{H}]$} & 0.065 & -0.020 & -0.001 & -0.098 & 0.050 & 0.070 \\
{$[\mathrm{O} / \mathrm{Fe}]$} & -0.095 & -0.181 & 0.000 & -0.081 & 0.080 & 0.129 \\
{$[\mathrm{Na} / \mathrm{Fe}]$} & 0.095 & -0.125 & 0.003 & -0.126 & 0.049 & 0.112 \\
{$[\mathrm{Mg} / \mathrm{Fe}]$} & 0.025 & -0.015 & 0.000 & -0.022 & 0.160 & 0.209 \\
{$[\mathrm{Si} / \mathrm{Fe}]$} & 0.030 & 0.005 & 0.001 & -0.027 & 0.094 & 0.124 \\
{$[\mathrm{Ca} / \mathrm{Fe}]$} & 0.075 & -0.100 & 0.000 & -0.200 & 0.158 & 0.242 \\
{$[\mathrm{Ba} / \mathrm{Fe}]$} & 0.056 & 0.181 & 0.006 & -0.169 & 0.181 & 0.260 \\
\hline
\end{tabular}
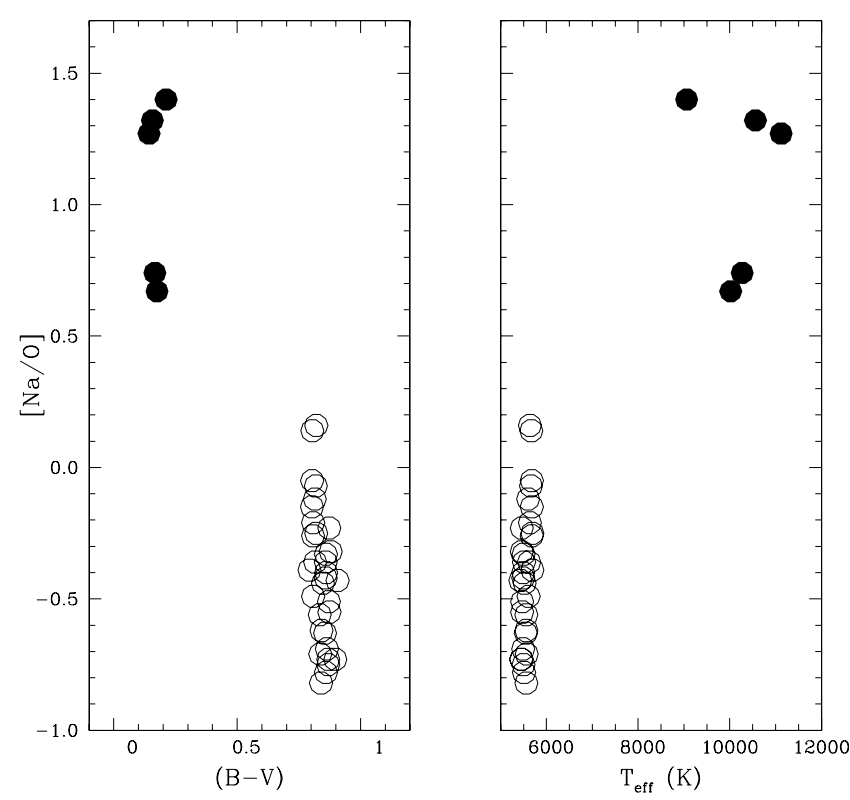

Fig. 4. Left: run of the $[\mathrm{Na} / \mathrm{O}]$ abundance ratio with $B-V$ colour along the HB of NGC 2808. Filled circles and open circles are for blue and red HB stars, respectively. Right: the same, but with $T_{\text {eff }}$ rather than colour.

we were unable to analyse the warmer stars along the $\mathrm{HB}$ of NGC 2808, we think this is a strong circumstantial confirmation of the scenario where the location of stars along the HB is determined also by their helium content. However, because there is a hint for a trend of $\mathrm{Na}$ and $\mathrm{O}$ abundances with colour also among the red HB stars alone, we suggest that there are more than three stellar populations in NGC 2808, and that the He-poor group itself divides into further groups. In order to produce the distribution in colours of the stars, the helium abundance differences among these groups should be small $(\leq 0.01)$. However, the presence of significant variations in $\mathrm{Na}$ content has implications in a scenario for the formation of this cluster.

\section{Conclusions}

We have obtained spectra for several tens stars on the HB of NGC 2808, which is the prototype mono-metallic, multiple stellar population cluster. Several red HB stars were observed, and six on the blue HB cooler than the gap at $11500 \mathrm{~K}$ studied by Grundahl, Caloi, and Behr. We found that as expected, the blue HB stars are O-poor and Na-rich, and the red HB ones are $\mathrm{O}$-rich and Na-poor. This strongly supports the identification of the $\mathrm{BHB}$ as the progeny of the intermediate MS, and of the RHB with the red MS, made by D'Antona et al. (2005, no reliable abundances could be obtained for the extremely blue HB stars, which should be the progeny of the blue MS stars). As additional information, we found that some Na-O anticorrelation can be found also among the RHB stars alone, suggesting that this sequence, which includes almost half of the HB stars in NGC 2808 itself, is made of at least two distinct groups of stars. Hence there are more than three stellar populations in NGC 2808.

Acknowledgements. This publication makes use of data products from the Two Micron All Sky Survey, which is a joint project of the University of Massachusetts and the Infrared Processing and Analysis Center/California Institute of Technology, funded by the National Aeronautics and Space Administration and the National Science Foundation. This research has made use of the NASA's Astrophysical Data System. This research has been funded by PRIN INAF "Formation and Early Evolution of Massive Star Clusters". We thank an anonymous referee for his/her useful suggestions.

\section{References}

Alonso, A., Arribas, S., \& Martinez-Roger, C. 1999, A\&AS, 140, 261 Alonso, A., Arribas, S., \& Martinez-Roger, C. 2001, A\&A, 376, 1039 Bedin, L. R., Piotto, G., Zoccali, M., et al. 2000, A\&A, 363, 159 Behr, B. B., Cohen, J. G., McCarthy, J. K., \& Djorgowski, S. G. 1999, ApJ, 517, L135

Behr, B. C., Djorgovski, S. G., Cohen, J. G., et al. 2000a, ApJ, 528, 849 Behr, B. C., Cohen, J. G., \& McCarthy, J. K. 2000b, ApJ, 531, L37 Bragaglia, A., Carretta, E., Gratton, R. G., et al. 2010a, A\&A, 519, A60 Bragaglia, A., Carretta, E., Gratton, R. G., et al. 2010b, ApJ, 720, L41 Caloi, V. 1999, A\&A, 343, 904

Cardelli, J. A., Clayton, G. C., \& Mathis, J. S. 1989, ApJ, 345, 245

Carney, B. W., Gray, D. F., Yong, D., et al. 2008, AJ, 135, 892

Carretta, E. 2006, AJ, 131, 1766

Carretta, E., Bragaglia, A., \& Cacciari, C. 2004, ApJ, 610, L25

Carretta, E., Bragaglia, A., Gratton, R. G., et al. 2006, A\&A, 450, 523

Carretta, E., Recio-Blanco, A., Gratton, R. G., Piotto, G., \& Bragaglia, A. 2007, ApJ, 671, L125

Carretta, E., Bragaglia, A., Gratton, R. G., et al. 2009a, A\&A, 505, 117

Carretta, E., Bragaglia, A., Gratton, R. G., et al. 2009b, A\&A, 505, 139

Carretta, E., Bragaglia, A., Gratton, R. G., et al. 2010, A\&A, 516, A55

Cottrell, P. L., \& Da Costa, G. S. 1981, ApJ, 245, L79

Dalessandro, E., Salaris, M., Ferraro, F. R., et al. 2011, MNRAS, 410, 694

D'Antona, F., \& Caloi, V. 2004, ApJ, 611, 871 
D’Antona, F., Caloi, V., Montalban, J., Ventura, P., \& Gratton, R. 2002, A\&A, 395,69

DAntona, F., Bellazzini, M., Caloi, V., et al. 2005, ApJ, 631, 868

Denisenkov, P. A., \& Denisenkova, S. N. 1989, A. Tsir., 1538, 11

Fabbian, D., Recio-Blanco, A., Gratton, R. G., \& Piotto, G. 2005, A\&A, 434, 235

Gratton, R. G., Carretta, E., Eriksson, K., \& Gustafsson, B. 1999, A\&A, 350, 955

Gratton, R. G., Bonifacio, P., \& Bragaglia, A. 2001, A\&A, 369, 87

Gratton, R., Sneden, C., \& Carretta, E. 2004, ARA\&A, 42, 385

Gratton, R. G., Carretta, E., Bragaglia, A., Lucatello, S., \& D’Orazi, V. 2010, A\&A, 517, A81

Grundahl, F., Catelan, M., Landsman, W. B., Stetson, P. B., \& Andersen, M. I. 1999, ApJ, 524, 242

Harris, W. E. 1974, ApJ, 192, L161

Harris, W. E. 1996, AJ, 112, 1487

Kinman, T., Castelli, F., Cacciari, C., et al. 2000, A\&A, 364, 102

Kraft, R. P. 1994, PASP, 106, 553

Kupka, F., Ryabchikova, T. A., Piskunov, N. E., Stempels, H. C., \& Weiss, W. W. 2000, Balt. Astron., 9, 590

Lambert, D. L., McWilliam, A., \& Smith, V. V. 1992, ApJ, 386, L685

Lind, K., Asplund, M., Barklem, P. S., \& Belyaev, A. K. 2011, A\&A, 528, A103

Lucatello, S., \& Gratton, R. G. 2003, A\&A, 406, 691
Magain, P. 1984, A\&A, 134, 189

Marino, A. F., Villanova, S., Milone, A. P., et al. 2011, ApJ, 730, L16

Mashonkina, I. I., Shimanskii, V. V., \& Sakhibullin, N. A. 2000, Astron. Rep., 44, 790

Momany, Y., Bedin, L. R., Cassisi, S., et al. 2004, A\&A, 420, 605

Norris, J., Cottrell, P. L., Freeman, K. C., \& Da Costa, G. S. 1981, ApJ, 244, 205

Osborn, W. 1971, Obs., 91, 223

Pace, G., Recio-Blanco, A., Piotto, G., \& Momany, Y. 2006, A\&A, 452, 493

Pasquini, L., Castillo, R., Dekker, H., et al. 2004, SPIE, 5492, 136

Peterson, R. C., Rood, R. T., \& Crocker, D. A. 1995, ApJ, 453, 214

Piotto, G. 2008, MSAIt, 79, 334

Piotto, G., Bedin, L. R., Anderson, J., et al. 2007, ApJ, 661, L53

Recio-Blanco, A., Piotto, G., Aparicio, A., \& Renzini, A. 2004, A\&A, 417, 597

Recio-Blanco, A., Aparicio, A., Piotto, G., De Angeli, F., \& Djorgovski, S. G. 2006, A\&A, 452, 875

Sandage, A., \& Wildey, R. 1967, ApJ, 150, 469

Skrutskie, M. F., Cutri, R. M., Stiening, R., et al. 2006, AJ, 131, 1163

Takeda, Y. 1997, PASJ, 49, 471

van den Bergh, S. 1967, AJ, 72, 70

Ventura, P., D’Antona, F., Mazzitelli, I., \& Gratton, R. 2001, ApJ, 550, L65

Villanova, S., Piotto, G., \& Gratton, R. G. 2009, A\&A, 499, 755

Villanova, S., Geisler, D., Piotto, G., \& Gratton, R. 2011, ApJ, submitted 